\title{
SUPERCONDUCTING WHEN WET
}

Ever since Jan Baptista van Helmont covered his willow-tree pot with an iron lid to prevent dust from contaminating his experiment - he wanted to weigh the change in soil mass as the plant grew, to deduce how much of the plant 'arose out of water only' - scientific experimenters have aimed for greater control of conditions, excluding the contaminants and contingencies of the wider world. And with good reason: it was surely contamination that kept the notion of spontaneous generation alive for so long, just as it continues to hamper studies of organic matter in meteorites. But without a serendipitous sprinkling of unforeseen ingredients, we might have been left waiting longer for antibiotics, dynamite, neoprene, perhaps even glass.

That's a moral reinforced by the recent discovery that superconductivity can be induced in strontium iron arsenide $\left(\mathrm{SrFe}_{2} \mathrm{As}_{2}\right)$ by water vapour ${ }^{1}$. The superconductivity of an iron oxypnictide (arsenide) compound was announced to great excitement in early 2008 by Hideo Hosono and his co-workers at the Tokyo Institute of Technology ${ }^{2}$. The interest stemmed from the transition temperature $\left(T_{\mathrm{c}}\right)$, which, at $26 \mathrm{~K}$, was warm enough to make this a highly promising materials system for producing new hightemperature superconductors. Sure enough, variants with $T_{\mathrm{c}}$ up to $54 \mathrm{~K}$ followed within weeks ${ }^{3,4}$.

The initial discovery was in fluorine-doped $\mathrm{LaFeAsO}$, a material with a layered crystal structure reminiscent of the copper oxide high$T_{\mathrm{c}}$ compounds. Some later versions dispensed with oxygen and replaced the rare-earth elements with alkali metals or alkaline earths; the record $T_{c}$ so far ( $56 \mathrm{~K}$ ) is in thallium-doped $\mathrm{GdFeAsO}$ (ref. 5). Pretty much all of them seem to have required doping by electrons or holes to show superconductivity.

That's where the new compound differs: there is no doping. Instead, Hosono and his colleagues ${ }^{1}$ found that a superconducting transition occurs at $25 \mathrm{~K}$ when water vapour diffuses into thin films. It's not an obvious ploy to try, and indeed they didn't plan to. They simply made the thin films by pulsed laser deposition and probed their conductivity in the normal way, unsurprised to find that the undoped material was not superconducting at all. But a few hours later, it was. The researchers realized that something in the ambient air must have caused the change. They ruled out oxygen, nitrogen and carbon dioxide before identifying water as the culprit.

This is not a wholly new phenomenon. Some other exotic, layered mixed-metal oxides and sulphides have been found to

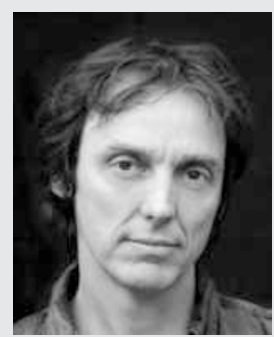

PHILIP BALL

superconduct when they intercalate water. But that is thought to happen because the water pushes the layers apart and increases the two-dimensional character thought to be central to this kind of superconductivity. On the contrary, the layers in $\mathrm{SrFe}_{2} \mathrm{As}_{2}$ get closer together when exposed to water, so something else is going on. Hosono and colleagues admit that they don't yet know what, but the answer might provide insights into the currently unknown superconducting mechanism. The effect could also take the transition temperatures of the iron pnictides to new heights.

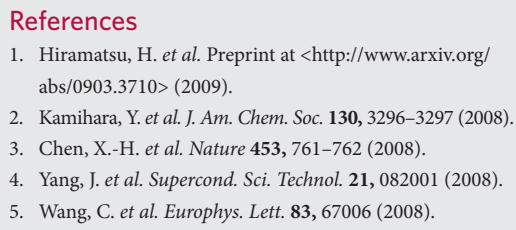

It's been possible for some time to create proteins synthetically, but cell-free gels that can produce proteins up to 300 times as efficiently as solution processes could provide new directions and greater complexity in synthetic biology.

\section{Kersten S. Rabe and Christof M. Niemeyer}

A lthough today's polymers are abundant in commercial products and indispensable in everyone's life, research efforts to improve material properties further and thus widen the scope of applications has never abated. Biopolymers - polymeric materials produced by living organisms - are of particular interest. Not only do these materials offer alternatives to synthetic polymers based on the ever-decreasing oil resources, but they can also possess biocompatibility, which is pivotal for many medical applications. Creating these biopolymers, such as proteins, outside the body and without using cells, has been possible in solution for some time. On page 432 of this issue, Dan Luo and colleagues add a new dimension to the process by constructing cell-free proteinproducing gels ${ }^{1}$. Their approach is far 